\title{
Full-length transcript amplification and sequencing as universal method to test mRNA integrity and biallelic expression in mismatch repair genes
}

\author{
Monika Morak $\mathbb{D}^{1,2} \cdot$ Kerstin Schaefer $^{1} \cdot$ Verena Steinke-Lange $^{1,2} \cdot$ Udo Koehler $^{2}$ - Susanne Keinath ${ }^{1}$.

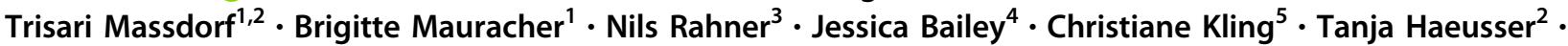 \\ Andreas Laner ${ }^{2} \cdot$ Elke Holinski-Feder $^{1,2}$
}

Received: 27 February 2019 / Revised: 13 May 2019 / Accepted: 2 July 2019 / Published online: 22 July 2019

(c) The Author(s), under exclusive licence to European Society of Human Genetics 2019

\begin{abstract}
In pathogenicity assessment, RNA-based analyses are important for the correct classification of variants, and require genespecific cut-offs for allelic representation and alternative/aberrant splicing. Beside this, the diagnostic yield of RNA-based techniques capable to detect aberrant splicing or allelic loss due to intronic/regulatory variants has to be elaborated. We established a cDNA analysis for full-length transcripts (FLT) of the four DNA mismatch repair (MMR) genes to investigate the splicing pattern and transcript integrity with active/inhibited nonsense-mediated mRNA-decay (NMD). Validation was based on results from normal controls, samples with premature termination codons (PTC), samples with splice-site defects (SSD), and samples with pathogenic putative missense variants. The method was applied to patients with variants of uncertain significance (VUS) or unexplained immunohistochemical MMR deficiency. We categorized the allelic representation into biallelic $(50 \pm$ $10 \%$ ) or allelic loss $(\leq 10 \%)$, and $>10 \%$ and $<40 \%$ as unclear. We defined isoforms up to $10 \%$ and exon-specific exceptions as alternative splicing, set the cut-off for SSD in cDNA $+\mathrm{P}$ to $30-50 \%$, and regard $>10 \%$ and $<30 \%$ as unclear. FLT cDNA analyses designated $16 \%$ of all putative missense variants and 12\% of VUS as SSD, detected MMR-defects in 19\% of the unsolved patients, and re-classified $>30 \%$ of VUS. Our method allows a standardized, systematic cDNA analysis of the MMR FLTs to assess the pathogenicity mechanism of VUS on RNA level, which will gain relevance for precision medicine and gene therapy. Diagnostic accuracy will be enhanced by detecting MMR defects in hitherto unsolved patients. The data generated will help to calibrate a high-throughput NGS-based mRNA-analysis and optimize prediction programs.
\end{abstract}

Supplementary information The online version of this article (https:// doi.org/10.1038/s41431-019-0472-8) contains supplementary material, which is available to authorized users.

Monika Morak

monika.morak@med.uni-muenchen.de

$\triangle$ Elke Holinski-Feder

elke.holinskifeder@med.uni-muenchen.de

1 Medizinische Klinik und Poliklinik IV, Campus Innenstadt, Klinikum der Universität München, Ziemssenstr. 1, 80336 Munich, Germany

2 MGZ - Medical Genetics Center, Bayerstr. 3-5, 80335 Munich, Germany

3 Medical Faculty, Institute of Human Genetics, Heinrich-Heine University, Düsseldorf, Germany

4 Clinical Genetics, St. George's University Hospital NHS Foundation Trust, London, UK

5 MVZ Labor Dr. Fenner und Kollegen, Hamburg, Germany

\section{Introduction}

Heterozygous germline defects in the four MMR genes MLH1, MSH2, MSH6, and PMS2 represent the genetic cause for Lynch Syndrome (LS), the most common hereditary predisposition for colorectal cancer (CRC) and associated tumors with autosomal dominant inheritance [1] showing a gene-specific difference in penetrance [2]. LS tumors typically display high microsatellite instability (MSI-H) and immunohistochemical (IHC) loss of the respective MMR protein. For $65 \%$ of the patients with suspicion of LS documented in the German HNPCC Consortium, the diagnosis is molecularly confirmed by the identification of a (likely) pathogenic MMR germline variant [3], according to the InSiGHT criteria [4]. Thirty-five percent of our patients fulfilling the clinical criteria of LS remain unsolved due to the detection of either a germline VUS (20\%), or no relevant variant at all $(15 \%)$ in the MMR genes [3,5]. For several 
sporadic cases, the accumulation of two somatic hits may serve as an explanation, but for those with familial tumor clustering the suspicion of a hereditary tumor syndrome remains [6]. Among VUS, missense variants can exert a functional defect on protein level, for which in silico prediction programs and in vitro assays are performed to define their pathogenicity [7-10], but they can also confer a splicing defect. Splice prediction programs are very reliable only for variants within the conserved splice sites [11, 12], but not for variants altering an exonic splice enhancer (ESE) or generating an exonic splice silencer as well as intronic motifs. Furthermore, the in vivo effect of SSD variants can be versatile and incomputable in silico. There is also growing evidence that several supposed missense variants in the MMR genes in fact generate a SSD [11, 13]. The same may be true for assumed synonymous variants, small indels [11, 13, 14], and even for truncating variants [15-17]. For a correct pathogenicity assessment of variants, an SSD should be excluded prior to functional analyses on protein level. The identification of SSD as a primary cause of disease implies the possibility of therapeutic approaches that target splicing $[18,19]$, and thus, a correct assessment of a variants' pathogenicity mechanism is mandatory.

The interpretation of cDNA analyses results, which are necessary to investigate the effect of VUS on mRNA level, is impeded by the presence of a variety of alternatively spliced isoforms of the MMR genes already reported in literature [20-23], which have to be discriminated from aberrant, allele-specific splicing [24, 25]. Alternative splicing (AS) is a common phenomenon occurring in $74 \%$ of the human multi-exon genes [26], but hitherto its functional relevance, if any, has only been speculated. For the four MMR genes, AS is regarded to result in no or nonfunctional proteins $[16,27]$. This implies that a variant-induced SSD in the MMR transcripts exceeding a certain threshold has to be considered as pathogenic, even for exons known for AS, and for isoforms with in-frame (if) deletions. This is different, e.g., for BRCAl and BRCA2, where splicing if can result in a functional protein, and is not regarded as pathogenic even with abundance of $50 \%$ of the transcripts $[28,29]$. The detection of aberrant splicing of MMR transcripts due to a VUS helps in pathogenicity assessment of that variant, especially when a complete, allele-specific SSD can be demonstrated. However, transcripts encoding an early PTC by a nucleotide change, deletion, frameshift, or SSD are subjected to degradation by NMD [30], and might barely be detected by RNA-based analysis. For pathogenicity assessment of variants on RNA level, we established a robust and universal protocol for cDNA analysis of the four MMR FLT by leukocyte culture, investigation of active/inhibited NMD by incubation without/with puromycin (cDNA-P/+P) [31], RT-PCR of the FLT and sequencing. We defined the normal AS pattern and biallelic expression in normal controls, and the cut-off values for SSD and allelic loss (AL) in samples with pathogenic variants generating an early PTC or conferring an SSD. The method was applied to samples with VUS or molecularly unsolved patients suspected of having LS.

\section{Materials and methods}

\section{Study cohort}

All patients meet at least the revised Bethesda criteria [32] and provided their informed consent for cancer genetics research compliant with ethical standards. We applied the recent InSiGHT guidelines [4] (2018-06_InSiGHT_VIC_v2.4 at https://www.insight-group.org/criteria/) to categorize variants as class 5 (pathogenic), class 4 (likely pathogenic), class 3 (VUS), class 2 (likely not pathogenic), or class 1 (not pathogenic).

Control cohort 1 included samples from patients with MMR-proficient tumors and normal germline testing of the four MMR genes. For each MMR transcript, ten sample pairs (cDNA-P/ $+\mathrm{P}$ ) were analyzed, and all had a heterozygous variant in the transcript (informative variant) in MLH1, MSH6, or PMS2 of class 1-2 (likely) benign. For $M S H 2$ coding variants are so rare that only three of the ten samples had an informative variant.

Control cohort 2 comprised 25 samples from patients with a (likely) pathogenic class $4-5$ variant (shown in Table 1 above), four of these patients additionally harbored a class 3 VUS.

Patient cohort 1 was composed of 22 patients with a variant of uncertain significance (21 different types) detected in one of the MMR genes (Table 1) including potential SSD variants, missense variants or deletions of one amino acid, synonymous variants, and an exon duplication ("class 3 variant group") to test the method on VUS.

Patient cohort 2 comprised 26 patients with deficiency for at least one MMR protein in IHC staining, no potentially causative MMR germline sequence alteration (classes 3-5) detectable ("with unsolved MMR-deficiency"), beside an informative variant (class 1) in the target MMR transcript. IHC deficiency was reported for MLH1/PMS2 in 16 patients, for PMS2 in 5 patients, for MSH2/MSH6 in 2 patients, and for MSH6 in 3 patients. Somatic MLH1 promoter methylation was excluded by MS-MLPA (MRCHolland) [33, 34] in 13 of 16 MLH1-deficient patients for which tumor DNA was available.

\section{cDNA preparation}

Lymphocytes were separated from human whole blood using LeucoSep tubes (Greiner Bio-One), cultured for 
Table 1 List of MMR gene variants analyzed with location given in consecutively numbered exon (e) or intervening sequence (IVS)

\begin{tabular}{|c|c|c|c|c|c|}
\hline Cohort & Variant types & $\begin{array}{l}\sum \text { patient } \\
\text { samples }\end{array}$ & MMR variant & $\begin{array}{l}\text { Class prior/ } \\
\text { posterior }\end{array}$ & $\begin{array}{l}\text { Effect on mRNA } \\
\% \text { splicing }\end{array}$ \\
\hline \multirow[t]{29}{*}{ Control cohort 2} & \multirow{12}{*}{$\begin{array}{l}\text { Early PTC (nonsense, } \\
\text { frameshift, exon deletion } \\
\text { oof) }\end{array}$} & \multirow[t]{12}{*}{10} & MLH1 e12 c. $1342 \mathrm{G}>\mathrm{T}$ p.(Glu448*) & 5 & $? 15 \%(+\mathrm{AL})$ \\
\hline & & & $\begin{array}{l}\text { MSH2 e2 c.242_243insA p. } \\
\text { (Ser81Argfs*2) }\end{array}$ & 5 & $-(\mathrm{AL})$ \\
\hline & & & MSH2 e7 c. $1147 \mathrm{C}>\mathrm{T}$ p. $\left(\operatorname{Arg} 383^{*}\right)$ & 5 & $-(\mathrm{AL})$ \\
\hline & & & MSH2 e7 c. $1165 \mathrm{C}>\mathrm{T}$ p. $\left(\operatorname{Arg} 389^{*}\right)$ & 5 & $-(\mathrm{AL})$ \\
\hline & & & MSH2 e7 c. $1215 \mathrm{C}>\mathrm{A}$ p. $\left(\mathrm{Tyr} 405^{*}\right)$ & 5 & $-(\mathrm{AL})$ \\
\hline & & & $\begin{array}{l}\text { MSH2 e8 c.1321dupA p. } \\
\text { (Thr441Asnfs*2) }\end{array}$ & 5 & $-(\mathrm{AL})$ \\
\hline & & & $\begin{array}{l}\text { MSH6 e4 c.1421_1422dup p. } \\
\text { (Gln47SCysfs*7) }\end{array}$ & 5 & $-(\mathrm{AL})$ \\
\hline & & & $P M S 2$ e6 c.697C>T p.(Gln233*) & 5 & $-(\mathrm{AL})$ \\
\hline & & & +VUS e13 c.2249G>A p.(Gly750Asp) & 3/in trans & - \\
\hline & & & $P M S 2$ e11 c. $1666 \mathrm{G}>\mathrm{T}$ p. $($ Glu556*) & 5 & $-(\mathrm{AL})$ \\
\hline & & & $\begin{array}{l}\text { PMS2 e11 c.(1144+1_1145-1)_(2006 } \\
\text { +1_2007-1)del }\end{array}$ & 5 & -v.g.del. \\
\hline & & & + VUS e13 c.2181G >C p.(Gln727His) & 3/in cis & - \\
\hline & \multirow[t]{3}{*}{$\begin{array}{l}\text { Late PTC (frameshift, exon } \\
\text { deletion oof) }\end{array}$} & \multirow[t]{3}{*}{3} & $\begin{array}{l}\text { MSH6 e9 c.3969_3979del p. } \\
\text { (Phe1323Leufs*14) }\end{array}$ & 5 & $\begin{array}{l}\text { SSD } 35 \%+\text { v.g. } \\
\text { del. }\end{array}$ \\
\hline & & & $\begin{array}{l}\text { PMS } 2 \text { e14 c.(2275+1_2276-1)_(2445 } \\
\left.+1 \_2446-1\right) \text { del }\end{array}$ & 5 & -v.g.del. \\
\hline & & & 2 cases & & \\
\hline & \multirow[t]{6}{*}{ Splice site variant } & \multirow[t]{6}{*}{6} & MLH1 IVS11 c.1039-2A>T & 4 & SSD $30 \%$ \\
\hline & & & MLH1 IVS13 c. $1558+1 \mathrm{G}>\mathrm{A}$ & $4 / 5$ & SSD $15 \%(+\mathrm{AL})$ \\
\hline & & & MLH1 IVS13 c. $1559-1 \mathrm{G}>\mathrm{C}$ & $4 / 5$ & SSD $50 \%$ \\
\hline & & & $M S H 2$ e 5 c. $942+3 \mathrm{~A}>\mathrm{T}$ & 5 & SSD $50 \%$ \\
\hline & & & $M S H 2$ e 16 c. $2635-1 \mathrm{G}>\mathrm{T}$ & 4 & SSD $50 \%$ \\
\hline & & & $P M S 2$ e8 c.903G > T p.(Lys301Asn) & $4 / 5$ & SSD $50 \%$ \\
\hline & \multirow[t]{8}{*}{$\begin{array}{l}\text { Missense variant, deletion of } \\
\text { one amino acid if }\end{array}$} & \multirow[t]{8}{*}{6} & $\begin{array}{l}\text { MLH1 e16 c.1852_1854del p. } \\
\text { (Lys618del) }\end{array}$ & 5 & - \\
\hline & & & MLH1 e4 c. $350 \mathrm{C}>\mathrm{T}$ p.(Thr117Met) & 5 & - \\
\hline & & & $M L H 1$ e10 c.793C > T p.(Arg265Cys) & 5 & SSD $45 \%$ \\
\hline & & & MLH1 e11 c.986A>C p.(His329Pro) & 5 & SSD $40 \%$ \\
\hline & & & MLH1 e11 c.986A>C p.(His329Pro) & 5 & SSD $30 \%$ \\
\hline & & & + VUS e2 c.191A >G p.(Asn64Ser) & $3 / 2$ in trans & - \\
\hline & & & MLH1 e17 c.1984A>C p.(Thr662Pro) & 4 & SSD $30 \%$ \\
\hline & & & + VUS e14 c.1653C>T p.(Asn551Asn) & $3 / 2$ in cis & - \\
\hline \multirow[t]{10}{*}{$\begin{array}{l}\text { Variants of uncertain } \\
\text { significance }\end{array}$} & \multirow[t]{3}{*}{ Potential splice site defect } & \multirow[t]{3}{*}{3} & $\begin{array}{l}\text { MLH1 IVS17 c.1989+4_1989 } \\
\text { +5insC p.? }\end{array}$ & $3 / 5$ & SSD $50 \%$ \\
\hline & & & MSH2 e 7 c. $1275 \mathrm{~A}>\mathrm{G}$ p. $(\mathrm{Glu} 425=)$ & 3 & SSD $35 \%$ \\
\hline & & & MSH6 IVS6 c.3556+3_3556+13del p.? & $3 / 5$ & SSD $50 \%$ \\
\hline & \multirow[t]{7}{*}{$\begin{array}{l}\text { Missense variant or in-frame } \\
\text { deletion of an amino acid }\end{array}$} & \multirow[t]{7}{*}{13} & $\begin{array}{l}\text { MLH1 e10 c.799_800delGTinsAG p. } \\
\text { (Val267Arg) }\end{array}$ & 3 & - \\
\hline & & & MLH1 e11 c.1013A>G p.(Asn338Ser) & 3 & - \\
\hline & & & MLH1 e13 c.1418A>G p.(His473Arg) & 3 & - \\
\hline & & & MLH1 e17 c.1964T>C p.(lle655Thr) & 3 & - \\
\hline & & & MLH1 e19 c. $2135 \mathrm{G}>\mathrm{C}$ p.(Trp712Ser) & 3 & - \\
\hline & & & MSH2 e2 c.319G>C p.(Ala107Pro) & 3 & - \\
\hline & & & MSH2 e8 c.1316_1318del p.(Pro439del) & 3 & - \\
\hline
\end{tabular}


Table 1 (continued)

\begin{tabular}{|c|c|c|c|c|c|}
\hline Cohort & Variant types & $\begin{array}{l}\sum \text { patient } \\
\text { samples }\end{array}$ & MMR variant & $\begin{array}{l}\text { Class prior/ } \\
\text { posterior }\end{array}$ & $\begin{array}{l}\text { Effect on mRNA } \\
\% \text { splicing }\end{array}$ \\
\hline & & & MSH2 e14 c.2399T>C p.(Leu800Pro) & 3 & - \\
\hline & & & $\begin{array}{l}\text { MSH6 e5 c.3264_3266del p. } \\
\text { (Phe1088del) }\end{array}$ & 3 & - \\
\hline & & & MSH6 e5 c.3286C>T p.(His1096Tyr) & 3 & - \\
\hline & & & MSH6 e8 c.3758T>A p.(Val1253Glu) & 3 & - \\
\hline & & & $P M S 2$ e11 c. $1437 \mathrm{C}>\mathrm{G}$ p.(His479Gln) & 3 & - \\
\hline & & & $P M S 2$ e 13 c. $2240 \mathrm{G}>\mathrm{C}$ p. $(\operatorname{Arg} 745 \mathrm{Thr})$ & 3 & - \\
\hline & Exon duplication & 2 & $\begin{array}{l}\text { PMS } 2 \text { e } 12 \text { c.(2006+1_2007-1)_(2174 } \\
\left.+1 \_2175-1\right) \text { dup r.? p.? }\end{array}$ & $3 / 4$ & -v.g.dup. \\
\hline & & & 2 cases & & \\
\hline & Synonymous variant & 4 & $M L H 1$ e 12 c. $1401 \mathrm{C}>\mathrm{T}$ p. $($ Ser467=) & $3 / 2$ & - \\
\hline & & & MSH2 e 10 c. $1560 \mathrm{~A}>\mathrm{G}$ p. $(\mathrm{Gly} 520=)$ & $3 / 2$ & - \\
\hline & & & MSH6 e1 c. $135 \mathrm{C}>\mathrm{T}$ p. $(\mathrm{Gly} 45=)$ & $3 / 2$ & - \\
\hline & & & MSH6 e4 c. $1914 \mathrm{~T}>\mathrm{G}$ p.(Leu638=) & $3 / 2$ & - \\
\hline
\end{tabular}

Control cohort 2 was selected upon the presence of a variant classified as 4-5 (likely) pathogenic according to the LOVD database/InSiGHT. Four cases additionally harbor a class 3 VUS indicated as $(+)$, for which the allelic position in cis or in trans was determined by cDNA analyses. The 21 MMR variants of uncertain significance (class 3) found in 22 patients represent patient cohort "class 3 variant group" for which we investigated the variants' effect on splicing and allelic expression in cDNA samples.

Prior/posterior indicates the classification according InSiGHT before and after cDNA analyses for re-classification, * not according to InSiGHT guidelines. The variants' effect on mRNA is summarized (- indicating no effect on splicing, ? effect of uncertain significance, SSD significant splice site defect, and maximum sum of isoform intensities observed in cDNA+P in \%, AL allelic loss, v.g.del. verified genomic deletion in cDNA, v.g.dup. verified genomic duplication in cDNA). PTC premature termination codon, oof out-of-frame, if in-frame. Variants in bold represent supposed missense variants which turned out to be splicing defects

$72-96 \mathrm{~h}$ at $37^{\circ} \mathrm{C}$, followed by incubation with puromycin for $5 \mathrm{~h}$ at $37^{\circ} \mathrm{C}[25,31]$, resulting in cDNA + P. Simultaneously, lymphocytes were cultured without the addition of puromycin and denominated as cDNA-P. Total RNA was extracted according to the QIAamp RNA blood mini kit (QIAGEN) including a DNase digestion. For many patients, RNA was also isolated using the PAXgene tubes and blood RNA extraction kit (PreAnalytics) [25]. For cDNA synthesis, $1 \mu \mathrm{g}$ of total RNA was subjected to the iScript Select cDNA synthesis kit (BioRad) and Oligo-(dT) $)_{18}$ primer following the manufacturer's instructions, but altered incubation temperature $\left(48^{\circ} \mathrm{C}\right.$ ) and extended time (75-90 min). A test-PCR for feasibility of cDNA amplification was carried out for $M L H 1$ exons 7-8, and showed absence of genomic contamination if the cDNA fragment (132 bp) was present in the agarose gel in absence of the genomic fragment (280 bp) [33].

\section{CDNA amplification and sequence analysis}

From 1-2 $\mu$ cDNA a long-range RT-PCR (LR-PCR) was carried out for the transcript of $M L H 1, M S H 2, M S H 6$, or $P M S 2$ with the forward primer located in the $5^{\prime}$ untranslated region (UTR) of the first exon, and the reverse primers in the $3^{\prime} \mathrm{UTR}$ of the last exon of the longest transcript (primers details in Supplemental Table 1). To enable allelic discrimination for as many samples as possible, we included the $M L H 1$ promoter variant c. $-93 \mathrm{G}>\mathrm{A}$ with an allelic frequency (AF) of 0.441 in Europeans, which made it necessary to establish the LR-PCR for $M L H 1$ for the isoform with the longest 5'UTR, as described in Genbank. Further benign variants with considerable AF located in the coding regions of $M L H 1, M S H 2, M S H 6$, and PMS2 (listed in Supplemental Table 2) were used to investigate the allelic representation (AR). For annotation we used the mRNA and DNA RefSeq NM_000249.3, NG_007109.2 for MLH1, NM_000251.2, NG_007110.2 for MSH2, NM_000179.2, NG_007111.1 for MSH6, and NM_000535.5, NG_008466.1 for PMS2, and exons were numbered according to their appearance.

The MLH1 transcript of $2.452 \mathrm{bp}$ is amplified from exon 1 to 19 c.-148_*33 with IProof High-Fidelity DNA Polymerase (BioRad), annealing temperature $61^{\circ} \mathrm{C}$ for $30 \mathrm{~s}$, extension time $1 \mathrm{~min} 20 \mathrm{~s}$ in 39 cycles. The $M S H 2$ transcript of $2.965 \mathrm{bp}$ is amplified from exon 1 to 16 c.-41_-*121 with IProof High-Fidelity DNA Polymerase (BioRad), annealing temperature $59^{\circ} \mathrm{C}$ for $30 \mathrm{~s}$, extension time $1 \mathrm{~min} 30 \mathrm{~s}$ in 39 cycles. The MSH6 transcript of $4.163 \mathrm{bp}$ is amplified from exon 1 to 10 c. $-37 * 43$ with PrimeSTAR GXL DNA Polymerase (TAKARA Clontech), annealing temperature $60{ }^{\circ} \mathrm{C}$ for $15 \mathrm{~s}$, extension time $4 \mathrm{~min} 30 \mathrm{~s}$ in 30 cycles. The 


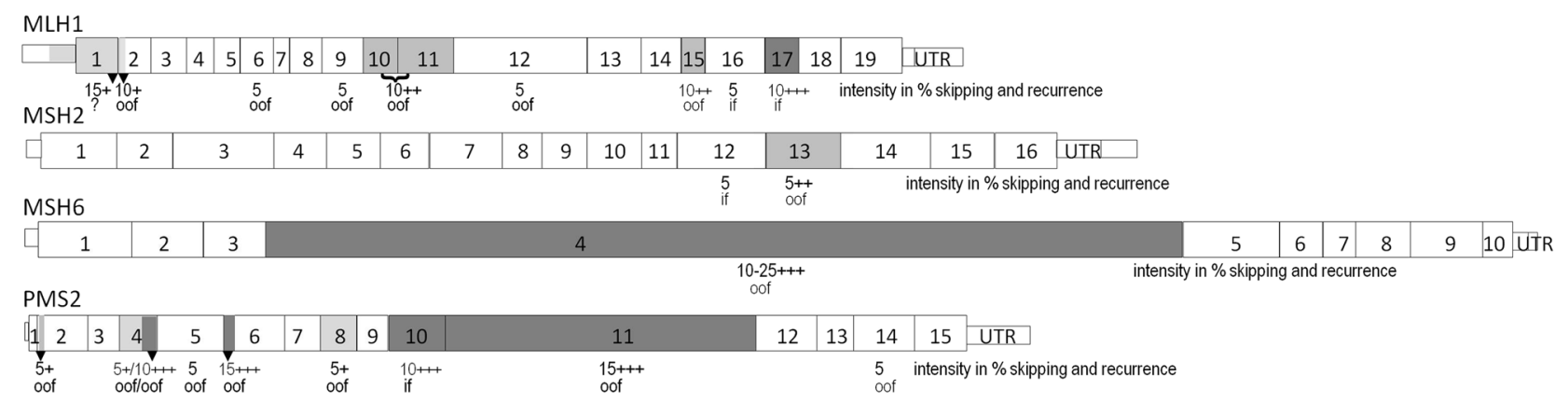

Fig. 1 Alternative splicing (AS) of a complete exon, a combination of two exons (with bracket), or a partial exon (black triangle) is depicted for the four MMR transcripts, as we detected it by LR-PCR in ten MMR-proficient control-pairs of cDNA-P/ $+\mathrm{P}$. The maximum relative intensity of $\mathrm{AS}$ in cDNA $+\mathrm{P}$ is given in \% below the exon number, the frequency how often AS was found in the 20 samples is indicated by $+/$ light gray for 1-3 samples, ++ /medium gray for recurrence in 4-6 samples, and +++/dark gray for frequent AS detected in seven or more samples, and the predicted effect of AS on the reading frame is given: if $=$ in frame, oof $=$ out-of frame
PMS2 transcript of $2.775 \mathrm{bp}$ is amplified from exon 1 to 15 c.-9_*177 with LA Taq DNA Polymerase (TAKARA Clontech) in a two-step PCR program with $68^{\circ} \mathrm{C}$ for $2 \mathrm{~min}$ $30 \mathrm{~s}$ in 30 cycles. In particular samples, additional short PCR amplifications were performed to investigate allelespecificity of a SSD or the cis/trans status of variants (primers see Supplemental Table 1). PCR products were visualized on a $1 \%$ agarose electrophoresis gel to check for successful FLT amplification, which was the case for 104 of 106 lymphocyte culture cDNAs-P/+P each, and for 26 out of 73 cDNAs from PAXgene indicating an insufficient quality of RNA from PAXgene tubes for FLT amplification. PCR products were treated with Exo-SAP kit (USB) and directly subjected to Sanger sequencing using several internal primers to generate overlapping reads of 600-900 bp length in one direction by using Big Dye v1.1 (Applied Biosystems). Sequences were run on ABI PRISM 3100 Avant.

Visual inspection of the FLT Sanger sequences was performed especially at known variants and exon-exon-junctions to assess the AR and the splicing pattern by calculation of relative intensities \% from the respective peak heights using Sequence Scanner v2.0 (Applied Biosystems)/Mutation Surveyor 3.1 (SoftGenetics) and rounded to intervals of 5. First, the AR of known heterozygous variants in the transcript (informative variants) was investigated in cDNA-P and cDNA $+\mathrm{P}$ in comparison to a wild-type (wt) sequence (examples in Supplemental Fig. 1A-D). The larger peak allele of a variant was defined as one allele representing $50 \%$, and the intensity of the other allele was calculated accordingly from the peak heights (Supplemental Fig. 1C, D). In a second step, the splicing patterns were assessed and compared between cDNA-P and cDNA $+\mathrm{P}$. Isoforms become visible by presence of an additional sequence usually starting at exon boundaries [25]. In sequences with two or three traces, we defined the wt, assigned the origin of additional sequences, and calculated intensities from their average peak heights (see Supplemental
Fig. 1E) with the assumption that the sum of FLT and AS isoform intensities is $100 \%$. Nomenclature is compliant with HGVS standard recommendations (http://varnomen.hgvs.org/ recommendations) referring to the genomic position in hg19. For SSD we mention the exon number for complete exon skipping, partial exon skipping of the $5^{\prime}$-end by $\mathrm{p}$, or $\mathrm{q}$ for skipping of the $3^{\prime}$ end, and intron/pseudo-exon inclusion as an insertion. We used the software Alamut Visual Version 2.10 (Interactive Biosoftware, France, http://www.interactivebiosoftware.com) to predict the variants' effect upon splicing (SpliceSiteFinder-Like, MaxEntScan, NNSPLICE, GeneSplicer, Human Splice Finder) and the variants' effect on protein function (Align GVGD, SIFT, Mutation Taster, MAPP, PolyPhen), evolutionary nucleotide conservation, and AF from the GnomAD browser or 1000 genomes. The cDNA results were submitted to the LOVD3 (https://databases.lovd. nl/shared/references/DOI:10.1038/s41431-019-0472-8).

\section{Results}

All results are based on cDNA from lymphocyte cultures enabling FLT analysis for $98 \%$ of the cDNAs, as PAXgene resulted in poor cDNA quality allowing FLT analysis in $36 \%$ of the cDNAs. Detailed results are documented in Supplemental Table 2, and summarized in Table 1 and Fig. 2.

\section{CDNA analysis in MMR-proficient samples (control cohort 1)}

The benign variants in ten sample pairs for $M L H 1, M S H 6$, and $P M S 2$, and three sample pairs for $M S H 2$ showed an AR with 40-55\% intensity (Supplemental Fig. 2A), so that biallelic expression was defined as $50 \% \mathrm{AR}$, allowing $10 \%$ standard deviation. AS was observed with an intensity of 5-10\% in cDNA-P for at least one isoform in 


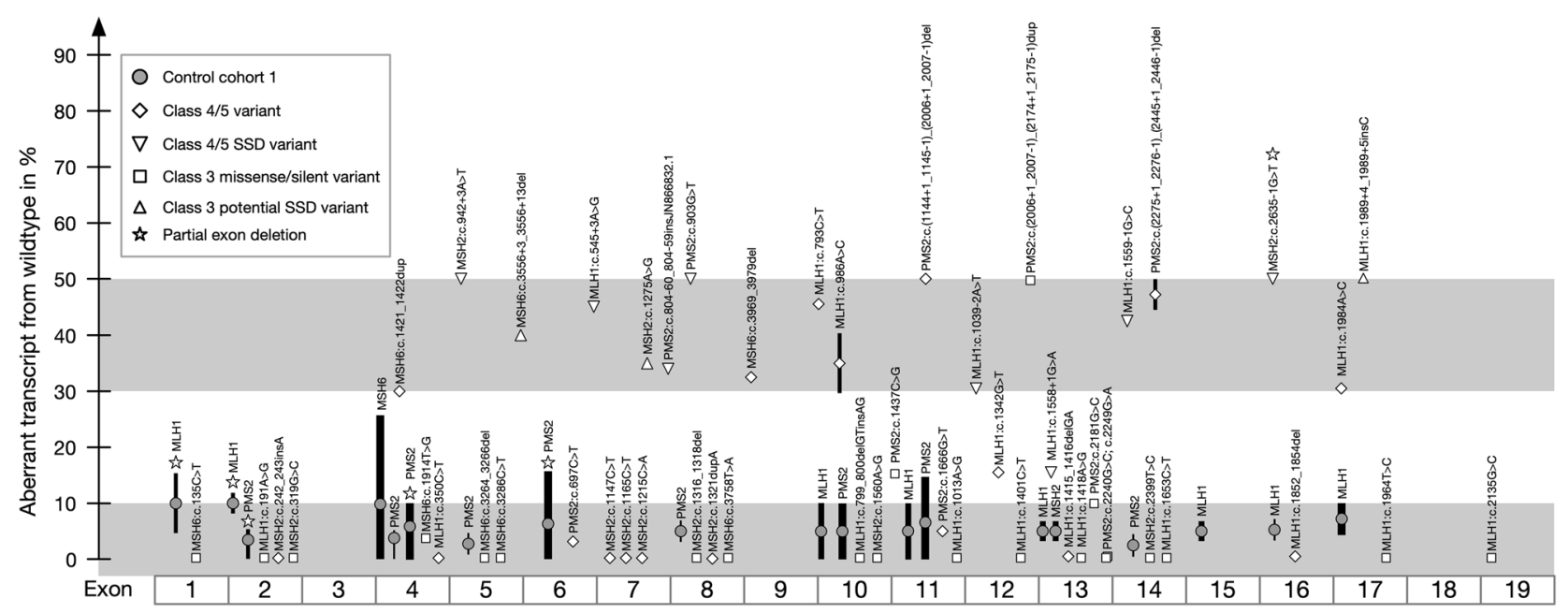

Fig. 2 Schematic presentation of splicing intensities in $\mathrm{cDNA}+\mathrm{P}$ found for variants in the four MMR transcripts $M L H 1, M S H 2, M S H 6$, and $P M S 2$ sorted by exon numbers. Exon skipping in normal controls (gray dots) was defined as alternative splicing, with their frequency correlated to the thickness of the line, the height giving the range of splicing intensity in $\%$. Alternative splicing usually ranged from $0 \%$ to $10 \%$, but showed a few exon-specific exceptions. Based on this, the

each MMR gene except for $M S H 2$. In cDNA+P, additional isoforms were present, and some exon-specific AS intensities reached up to $15 \%$ for $M L H 1$ or $P M S 2$, and up to $25 \%$ for MSH6. We found AS skipping of a single exon, two neighboring exons, or a part of an exon, for details see Fig. 1.

\section{CDNA analysis of samples with pathogenic variants (control cohort 2)}

\section{Nonsense-mediated decay in class 4-5 early and late PTC variants}

For each of the four major MMR transcripts at least one early PTC was investigated $(1 \times M L H 1,5 \times M S H 2,1 \times$ $M S H 6,3 \times P M S 2$, for details see Table 1$)$. The ten cDNAs$\mathrm{P}$ showed an almost monoallelic expression of the wt, whereas the early PTC was present with $0-10 \%$ intensity due to active NMD and defined as AL. In cDNAs+P the early PTC allele was restored, showing intensities of $15 \%-40 \%$ for eight of the ten variants (Supplemental Table 2) including aberrant splicing for one sample (Table 1, Fig. 2). Two of the five early PTC in $M S H 2$ were only visible with maximum $5 \%$ or $10 \%$ intensity in forward and reverse sequences despite NMD-inhibition, and no additional aberrant splicing was detected. For these two samples with $M S H 2$ variants in exon 2 (c.242_243insA p. (Ser81Argfs*2)) and exon 7 (c.1215 C >A p. (Tyr405*)) additional short PCRs from cDNA $+\mathrm{P}$ spanning exons $1-4$, or 4-12 and 5-8 showed the same underrepresentation of lower, light gray zone comprises splice-neutral variants. In samples with a designated splice site defect aberrant splicing was expected and found in a range of $30-50 \%$. The gray zone above was defined to designate splicing defects. After definition of the cut-off values, we investigated the splicing of the variant-bearing exon in samples with a PTC, pathogenic missense variant, or VUS including potential SSD, predicted missense or synonymous variant

the early PTC allele as in LR-PCR, making an AL due to primer selection unlikely. We tested NMD evasion on three sample pairs with late PTC variants in MSH6 or PMS2 which were not subjected to NMD, and detected aberrant splicing for one sample (Supplemental Table 2, Table 1, Fig. 2).

\section{Aberrant splicing in class 4-5 splice site variants}

To define cut-off values for aberrant splicing resulting in a $\mathrm{SSD}$, we analyzed cDNAs with six variants designated as class $4 / 5$ due to assumed SSD. Aberrant transcripts out-offrame (oof) generating an early PTC had intensities of $0-10 \%$ in cDNA-P due to NMD, whereas aberrant transcripts if showed $50 \%$ intensity.

MSH2 c. $942+3 \mathrm{~A}>\mathrm{T}$ and $P M S 2$ c. $903 \mathrm{G}>\mathrm{T}$ exerted a complete skipping of the affected exon with $50 \%$ intensity in $\mathrm{cDNA}+\mathrm{P} . \mathrm{MLH} 1 \mathrm{c} .1039-2 \mathrm{~A}>\mathrm{T}$ and $M L H 1 \mathrm{c} .1559-1 \mathrm{G}>\mathrm{C}$ created several isoforms in cDNA+P (in total 30-50\% intensity) skipping the affected exon and also neighboring exons. $M S H 2$ c. $2635-1 \mathrm{G}>\mathrm{T}$ induced a deletion of the first four nucleotides of exon 16 (r.2635_2638del p. Gln879Valfs*12) with $50 \%$ intensity. MLH1 c.1558 $+1 \mathrm{G}>\mathrm{A}$ inserted a part of intron 13 and enhanced AS of exons $9-10$ with $15 \%$ intensity each. The informative variant c.655A $>\mathrm{G}$ in this sample showed only $15-20 \%$ intensity in $\mathrm{cDNA}+\mathrm{P}$ for the $\mathrm{G}$ allele, indicating a reduced $\mathrm{AR}$ of the pathogenic allele and explaining the low percentage of aberrant splicing of $15 \%$. In InSiGHT database, another variant $M L H 1$ c. $1558+1 \mathrm{G}>\mathrm{T}$ was designated as class 5 
generating the same SSD with unknown intensity. Detailed information about the different SSD is given in Table 1 and Supplemental Table 2.

Taken together, the AR in a heterozygous variant is found with relative intensities of $40-60 \%$ in normal controls. The detection of a reduced AR of an informative variant $(<40 \%)$ indicates an abnormality so that potential splice site aberrations may not be assessed accordingly and needs further investigation. AS occurs with intensities up to $10 \%$ and exceeds this value for only few specific exons. Transcripts with an early PTC were underrepresented with $0-10 \%$ in cDNA-P due to NMD and defined as AL, but can be restored up to $15-40 \%$ intensity in cDNA $+\mathrm{P}$ by NMDinhibition in most of the cases. Variants in the conserved splice sites can reliably be predicted as SSD, and aberrant splicing was visible with 30-50\% intensity at least in cDNA $+\mathrm{P}$, or $15 \%$ in combination with a reduced AR (Fig. 2, Supplemental Table 2).

\section{class 4-5 missense variants or deletions of one amino acid}

Five missense variants assumed to affect one amino acid and already classified as pathogenic were analyzed in cDNA to test for the mechanism of pathogenicity. $M L H 1$ c.793C $>$ T p.(Arg265Cys) and MLH1 c.986A $>$ C p. (His39Pro) both created several isoforms by skipping different exons with aberrant transcript intensities from $30 \%$ to $45 \%$ in cDNA $+\mathrm{P}$, and the variant conferring the SSD was absent in the transcript (0-5\% intensity). The location of the variant MLH1 c. $191 \mathrm{~A}>\mathrm{G}$ p.(Asn64Ser) in trans to a pathogenic variant in the absence of constitutional mismatch repair-deficiency (CMMRD) features allows a re-classification of this VUS to class 2. MLHI c.1984A >C p.(Thr662Pro) showed 20\% intensity (reduced AR), and an if skipping of exon 17 with 15-30\% intensity. As exon 17 also shows AS, we checked for an aberrant allele-specific splicing by analyzing the VUS c. $1653 \mathrm{C}>\mathrm{T}$ p.(Asn551Asn) in $M L H 1$ exon 14 additionally present in the sample, which showed biallelical expression in cDNA-P/+P in the FLT, but a reduced AR of $20 \%$ intensity when selecting for the presence of exon 17 . MLH1 c.1852_1854del p.Lys618del and c.350C $>$ T p. Thr117Met showed an AR of 50\% intensity in cDNA-P/ $+\mathrm{P}$ in the absence of aberrant splicing, and are designated as splice-neutral. Detailed information about the different aberrant transcripts is given in Table 1 and Supplemental Table 2. In conclusion, three $M L H 1$ class 4-5 putative missense variants are in fact SSD (Fig. 2, Supplemental Fig. 2) even though not located next to a splice site, and not predicted as SSD by bioinformatics. The FLT analysis furthermore determined four VUS as splice-neutral, and their allelic status towards the pathogenic variant in the transcript (Table 1).

\section{CDNA analysis for class 3 MMR variants (patient cohort 1)}

By applying our FLT analysis method, we investigated the effect of further 21 different class 3 VUS from patient cohort 1 (Table 1) upon mRNA splicing (results depicted in Fig. 2, Supplemental Fig. 2, Supplemental Table 2):

\section{Potential SSD variants}

Three VUS potentially exert an SSD, as they are located close to a splice site, show an evolutionary sequence conservation of $62-84 \%$, and two to five algorithms predict a reduction/loss of the splice donor site. $M L H I$ c.1989+4_1989+5insC generated an exon 17 if skipping (r.1897_1989del p.Glu633_Glu663del) with 50\% intensity in cDNA-P/ $+\mathrm{P}$, and biallelic expression of the benign variant c. $1217 \mathrm{G}>$ A p.Ser406Asn in exon 12. An allelespecific $100 \%$ SSD was shown by amplification of exons 11-17 selecting for transcripts including exon 17 , where the allele c.1217A was monoallelically present. MSH2 c. $1275 \mathrm{~A}>\mathrm{G}$ p. $(\mathrm{Glu} 425=)$ induced an if skipping of the rear part of exon 7 (r.1229_1276del p.Ile411_Gly426del) with $30-35 \%$ intensity in cDNA-P/ $+\mathrm{P}$. The c.1275G allele was still present with $15 \%$ intensity, suggesting an allele-specific but incomplete SSD. The cDNA-P sample of MSH6 c. $3556+3 \_3556+13$ del showed an $\mathrm{AL}$ in a benign variant in exon 1 due to NMD. In cDNA $+\mathrm{P}$ a $50 \%$ skipping of exon 6 oof (r.3439_3556del p.Ala114Valfs*9) was detected, and the benign variant c.116G $>$ A p.Gly39Glu was biallelically represented with $50 \%$. To sum up, we found aberrant splicing with intensities of $30-50 \%$ for all three potential SSD VUS. Two variants were designated as complete SSD, which allowed a re-classification to class 5 (pathogenic), whereas the third variant showed a significant but partial SSD and remained class 3 .

\section{Missense variants or small in-frame deletions}

Based on a biallelic AR (45-60\%) and absence of aberrant splicing in cDNA-P/+P for the remaining 11 missense variants and two variants deleting one amino acid (Table 1), we designated these VUS as splice-neutral. However, for reclassification of these variants the effects on protein level have to be clarified in a consented manner.

\section{Exon duplication}

The FLT analysis of two samples with a genomic duplication in PMS2 exon 12 of unknown localization showed an if insertion r.2007_2174dup p.Lys670_Ala725dup with 50\% intensity in cDNA-P/ $+\mathrm{P}$ verifying the aberration of one allele in the PMS2 transcript. Even though InSiGHT rules 
would conclude class 3 for this if insertion, we regard it as class 4 because it occurs in the protein domain of functional importance for MLH1 interaction. Furthermore, their offspring with homozygous exon duplication shows CMMRD features and IHC loss of the PMS2 in normal and tumor tissue.

\section{Synonymous variants}

Four synonymous variants showed a biallelic expression with intensities of $50-60 \%$ in the absence of aberrant splicing. The AS was maximum 5\% for the exon harboring the variant or any exon in cDNA-P/ $+\mathrm{P}$, even for the variant located in MSH6 exon 4, for which elevated levels of AS was observed in controls. Our designation of these four synonymous variants $M L H 1$ c. $1401 \mathrm{C}>\mathrm{T}$ p.Ser $467=, M S H 2$ c. $1560 \mathrm{~A}>\mathrm{G}$ p.Gly520=, MSH6 c.135C $>\mathrm{T}$ p.Gly45=, c.1914T $>$ G p.Leu638 = as splice-neutral enabled a reclassification from class 3 to class 2 (Table 1).

\section{CDNA analysis in patients with unsolved MMR- deficiency (patient cohort 2)}

By investigating the AR of a benign, heterozygous variant (class 1) in the relevant FLT of MLH1, MSH2, MSH6, or $P M S 2$ we performed a screening for an $\mathrm{AL}$ or $\mathrm{SSD}$, which revealed an AR ranging between $45 \%$ and $55 \%$ and a normal splicing pattern in cDNA-P/+P for 21 of the 26 patients with unresolved MMR-deficient tumors. In cDNA$\mathrm{P}$ of five patients, an AL was detected with intensities of one allele below $10 \%$, four indicating a germline defect in $M L H 1$, and one in PMS2. In cDNA $+\mathrm{P}$ of three of these patients the NMD-inhibition restored the missing allele with an AR of $30-50 \%$ in benign variants. Furthermore, the explanatory pathogenic cDNA changes were visible in cDNA+P sequencing: an early PTC variant in $M L H 1$ exon 13 (r.1415_1416delGA p.Arg472Thrfs*6), an oof skipping of $M L H 1$ exon 6 (r.454_545del p.Thr152Leufs*3) with $45 \%$ intensity, and a $P M S 2$ insertion of 71 nucleotides prior to exon 8 (r.803_804insAATGTGCCATGTGAACC ACCCCGTCTGAAAAGTGAGGAGCCCCTCTGCCCG GCAGCCGCCCCGTCTGGGAG p.Tyr268*) with $35 \%$ intensity (Table 2, Fig. 2). Genomic re-sequencing of these patients revealed two pathogenic germline variants, which were initially overlooked in earlier routine diagnostics screening: the early PTC MLHI c.1415_1416delGA p.Arg472Thrfs*6 has not been detected by DHPLC mutation screening performed formerly [35], and the splice site variant MLH1 c. $545+3 \mathrm{~A}>\mathrm{G}$ explanatory for exon 6 skipping was only visible in the forward, but not in the reverse Sanger sequence and was therefore not noticed even by the mutation detection program. A large genomic SVAinsertion of $2.2 \mathrm{~kb}$ in PMS2 c.804-60_804-59insJN866832.1

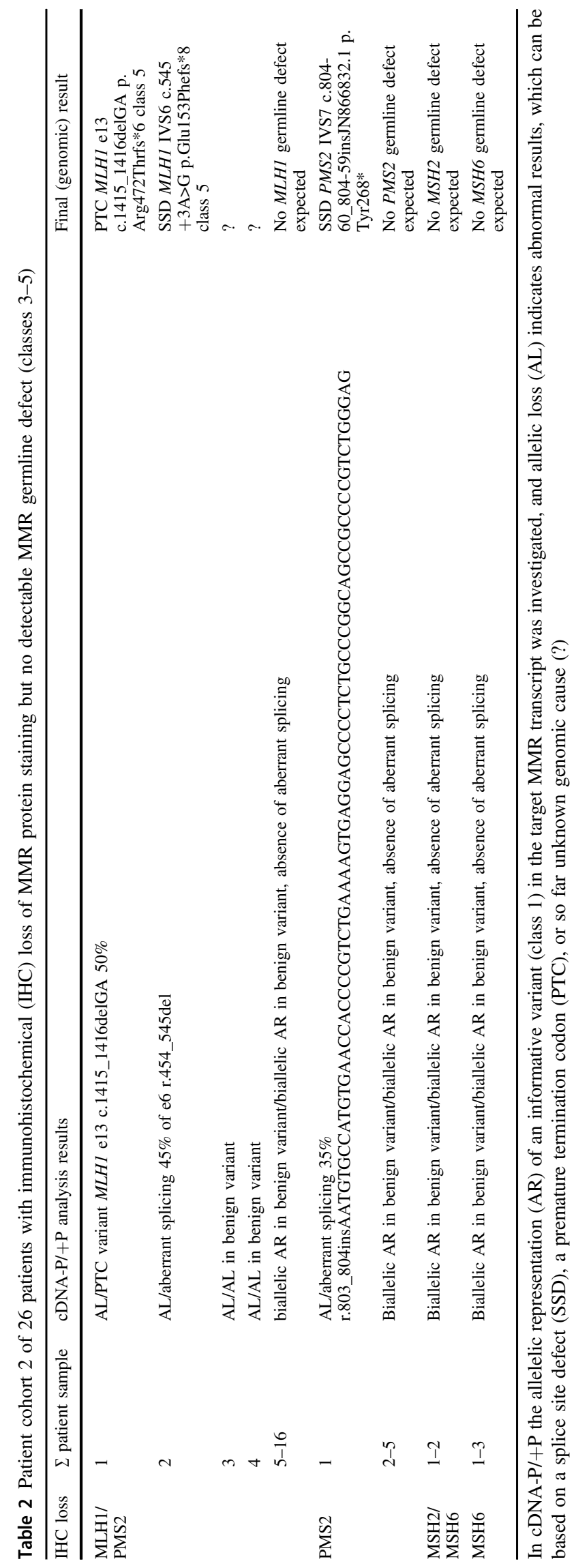


described previously [36] was found as the underlying genetic cause for the insertion oof on cDNA level. This insertion is located in the intronic region of exon 7 which was not analyzed in routine diagnostics at that time. In cDNA $+\mathrm{P}$ of two patients the AL of $M L H 1$ remained unsolved, and was confirmed in new, independent blood samples, but the reasons for this still need to be discovered.

\section{Discussion}

\section{Method standardization}

We comprehensively describe a robust protocol for the FLT analysis of the four major MMR genes MLH1, MSH2, MSH6, and PMS2 from patients' heparin blood samples in short term lymphocyte culture with/without puromycin incubation prior to RNA isolation, cDNA synthesis, LRPCR amplification of the FLT, and Sanger sequencing. We chose direct sequencing to avoid loss of low-abundance isoforms from gel-extraction, as previously described [37]. Lymphocyte culture at short term did not alter expression/ splicing in the MMR genes and has proven to be well suitable for FLT cDNA analysis. Even though the relative intensity of alleles and isoforms from Sanger sequences is semi-quantitative, we show that this method reliably allows the designation of a variants' expression to assess its effect on splicing and RNA-stability/integrity.

This is the first report of a FLT PCR protocol at least for MSH6 and PMS2. Previously, FLT amplification and analysis was published for $M L H 1$ and $M S H 2$ [13, 37-40], but did not cover the frequent variant $M L H 1$ c. $-93 \mathrm{G}>\mathrm{A}$, tested for an AL without sequencing the FLT [40], performed short PCRs and subcloning in a second step before sequencing [39], or performed sequencing with primers of variable orientation [37, 39, 41]. Most cDNA analyses were performed by RT-PCR amplifying only the patients' cDNA in a selected region of interest [7, 11, 15, 42]. Thereby, splicing might be overestimated because shorter isoforms can be preferentially amplified, effects only visible in the FLT such as intergenic inversions might be lost, and different results may be obtained regarding the extent of an SSD due to primer design. Minigene assays examine the inclusion of one specific exon containing a variant in an artificial sequence context in comparison to the wt in a monoallelic ex vivo environment using transfected cell lines. This technique has shown a high concordance to patients' cDNA analysis, but may in some cases differ in the extent and completeness of an SSD, which is depending on the usage of different vectors and types of cell line $[12,13,43]$. Minigene assays are very useful to assess the effect of variants including intronic variants, when patient RNA is not available, and to confirm an unexpected SSD.
We suggest the FLT cDNA analysis as the preferred method to investigate the effect of MMR variants upon splicing in vivo. Within the European Mismatch Repair Working Group we agreed on this FLT protocol to be able to compare cDNA results from different laboratories, and aim to achieve a greater analytical sensitivity and data consistency, as it was shown for cDNA analyses of BRCAl and BRCA2 [44].

\section{Assessment of biallelic expression and normal AS in controls}

Our first step in cDNA analyses was to investigate the AR in heterozygous variants in $\mathrm{cDNA}-\mathrm{P} /+\mathrm{P}$, which ranged 40-60\% relative intensity. The AS for all MMR transcripts was set at an intensity of $5-10 \%$, and some exon-specific exceptions were defined with intensities up to $15 \%$ in $M L H 1$ exon 1q, or PMS2 exon 6p and exon 11, and up to $25 \%$ for MSH6 exon 4 especially in cDNA $+\mathrm{P}$, where transcript degradation was blocked. These higher values of AS for specific exons may be due to a weaker splice site strength, influence of cis-regulatory elements, presence of alternative splice sites at other sites than exon boundaries, and for the large exons PMS2 exon 11 and MSH6 exon 4 a PCR bias preferentially amplifying shorter fragments may be possible explanations. Other values of splicing above $15 \%$ necessitate further examination including allelespecific underrepresentation. This AS reflects the profile in cultured blood lymphocytes and may also be specific for this method (Fig. 1) [25]. Even though many MMR isoforms are reported as AS [27], we reported only those with $\geq 5 \%$ intensity, and selected against isoforms with an alternative start or alternative end with our method by locating primers in the first and last exon in the wt FLT. In each MMR transcript, we detected at least one AS isoform with 5-10\% intensity. For $M L H 1$ several known isoforms were found, and one new isoform lacking exon 1q (r.-62_116del p.?), which may be due to the investigation of the transcript with the longest 5'UTR. In previous MLH1 FLT analyses, no relevant AS was described [38], or specific sequencing primers were used to select against AS [37]. For $M S H 2$ we recorded AS of exon 12 and 13 also described in partial cDNA analyses [45, 46]. In MSH6 none of the few alternative isoforms reported [27] showed significant intensity, but we frequently observed skipping of the entire exon 4, which to our knowledge was not described before [38]. For $P M S 2$ three isoforms were novel to our knowledge (skipping exons 8,11 , and 14), and partial splicing of exon $4 \mathrm{q}$ and $6 p$ already reported as AS was confirmed [38]. In previous RT-PCR cDNA studies mostly analyzing only parts of the MMR transcripts, the intensity of AS was rarely quantified, but described as significantly lower compared to the wt [22]. For MLH1 and MSH2 AS was measured with 
intensities up to $28 \%$ by subcloning [39]. Higher intensities of AS in partial cDNA analyses of MMR genes [20-22, 27], e.g., ranging from $20 \%$ to $60 \%$ for $M L H 1$ exons 15 and 16 [47] may potentially be overestimated due to a PCR bias for shorter fragments [25]. On the other hand, AS is reported to vary among individuals and tissues [22, 38, 47].

\section{NMD inhibition}

Late PTC variants had a biallelic AR. All MMR transcripts harboring an early PTC had an AR of only $0-10 \%$ in cDNA-P due to NMD, which we categorized an AL. Early PTC transcripts could be restored in cDNA+P with $15-40 \%$ intensity for $80 \%$ of the samples, whereas caution is advised for $\mathrm{MSH} 2$ where only $5 \%$ or $10 \%$ intensity may be obtained. In literature, some early PTC alleles in $M S H 2$ could also be restored to only $12 \%$ by NMD inhibition [39]. The usage of an alternative poly-adenylation signal abrogating amplification with the primers used, mRNA instability, or a mechanistic incomplete NMD inhibition for MSH2 might be an explanation. An elevated AS was observed for some pathogenic variants, but its clinical relevance remains uncertain $[11,27]$. Some PTC variants induce a partial SSD, which may be relevant for pathogenicity assessment, if the allele is rescued by an if deletion [15-17].

\section{Assessment of aberrant splicing}

Splice site variants generated isoforms with $30-50 \%$ intensity in at least $\mathrm{cDNA}+\mathrm{P}$, indicating a fairly complete SSD. As an exception, one sample showed an intron retention in $M L H 1$ with only $15 \%$ intensity combined with a reduced AR of $15-20 \%$. Here, either amplification of the larger isoform was less efficient, additional larger isoforms may not be amplified, or NMD-inhibition was insufficient. In literature, aberrant splicing was defined for partial cDNA analyses by the detection of an isoform intensity within the range of 35-60\% [39], or three-fold higher than AS [48]. However, we found that NMD-inhibition may restore only $15 \%$ of the PTC alleles. Therefore, also slightly elevated levels of isoforms may need further investigation, especially when combined with an allelic reduction below $25 \%$ intensity [49-51].

\section{Analysis of class 4-5 variants}

We designated MLH1 c.793C $>\mathrm{T}$ p.(Arg265Cys) as a complete SSD in cDNA affecting exon 10 and neighboring exons, which is in concordance with a report in (LOVD3 microattribution ID 200068 by Leung), and is supported by the proof of an ESE disruption [43], whereas only a partial splicing defect skipping exon 10 was described in minigene assays [13, 43]. Previously, for this variant a decreased protein stability [52] and reduced MMR efficiency [10] were reported. For variant $M L H 1$ c. $986 \mathrm{~A}>\mathrm{C}$ p.(His329Pro) classified as pathogenic by segregation in LOVD, we reported a complete SSD, whereas functional assays for the predicted protein indicated pathogenicity on protein level [9, 53]. For variant MLH1 c. $1984 \mathrm{~A}>\mathrm{C}$ p.(Thr662Pro) we found a clear but partial SSD with $30 \%$ aberrant splicing, described previously [42], and no evidence for an ESE disruption [43]. Two $M L H 1$ variants c.1852_1854del p.Lys618del and c.350C $>\mathrm{T}$ p.Thr117Met were splice-neutral, and protein instability and MMR impairment have been shown in functional protein assays as the underlying mechanism $[54,55]$. Protein predictions revealed a diseasecausing effect by three or five of five algorithms for $M L H I$ e17 c.1984A $>$ C p.(Thr662Pro) or MLH1 c.793C $>$ T p.(Arg265Cys) without any hint towards a possible SSD pointing out that missense variants generating an SSD cannot be reliably predicted. Therefore, transcript analysis of variants should ideally precede functional protein tests. Beside this functional misclassification, machine learning systems like in silico prediction tools for splicing and protein function are based on wrong information.

\section{Method application for VUS and patients with unsolved MMR deficiency}

Within this small number of 25 different class 3 VUS, we designated $12 \%$ of them $(3 / 25)$ as $\mathrm{SSD}$, which is in concordance with one report [12], while a percentage of $25 \%$ SSD amongst missense variants was reported elsewhere [11]. By FLT analysis, we were able to re-classify nine VUS, six as class 2 , and three as class $4 / 5$ allowing a clinical relevant interpretation. One variant was an incomplete SSD, the other 15 variants alter an amino acid and remain uncertain on protein level. The analysis of FLT in 26 patients with unsolved MMR deficiency showed abnormal results for five cases, presenting an $\mathrm{AL}$ for $P M S 2$ in one, and for $M L H 1$ in four cases in cDNA-P. NMD inhibition restored the missing allele in three patients, which were then solved by the detection of genomic pathogenic variants overlooked/not analyzed previously. The underlying cause of the AL of MLH1 in two patients could not be defined in the scope of this work, but was regarded as sufficient proof for a gene defect (LS) potentially based on a regulatory or structural defect.

\section{Conclusion}

By using the FLT MMR analysis in cDNA-P/+P we found $3 / 19$ putative missense variants (16\%) and 1/12 PTC variants $(8 \%)$ to unexpectedly exert an SSD, even 
though pathogenicity on protein level was assumed. Of all 25 class 3 VUS of different types, 3 were SSD (12\%) and 9 could be re-classified (36\%). Furthermore, the method detected a germline defect in five of the 26 patients with unsolved MMR-deficiency (19\%). In regard of the need for precision medicine, this method will help to elevate diagnostic accuracy in several regards: first, in finding regulatory or exonic variants missed by routine sequencing or previous screening. Second, the RNAbased pathogenicity assessment of class 3 missense or synonymous variants helps to identify SSD. Third, the correct definition of the mutation mechanism of pathogenic variants, which may constitute SSD, will become important in the context of gene therapy. And fourth, the data will help the education of prediction programs by enabling bioinformaticians to work with correct information. Being aware of the existence of highly homologous pseudogenes, our cDNA analysis for the PMS2 FLT rules out a co-amplification of pseudogenes, allowing the verification of pathogenic PMS2 variants, exon deletions/duplications, and the detection of gene conversions between PMS2 and PMS2CL [56]. As a limitation, our method may select against transcripts with large intron retention, duplication of several exons, alternative start or end and fusion transcripts due to our PCR design, which may show up as AL. As this method is tedious, timeconsuming and needs a very skilled laboratory, a great achievement of this work is the generation of prerequisite data that will be used in a next step to calibrate highthroughput NGS-based mRNA assays also necessitating cut-offs for biallelic/monoallelic expression and alternative/aberrant splicing.

Acknowledgements We thank all patients for their participation in this study, as well as their respective doctors for contributing materials and clinical information.

Funding This work was based on projects funded by grants from the German Cancer Aid (Deutsche Krebshilfe) (\#111222) and the Wilhelm Sander-Stiftung (\#2012.081.1).

\section{Compliance with ethical standards}

Conflict of interest The authors declare that they have no conflict of interest.

Publisher's note: Springer Nature remains neutral with regard to jurisdictional claims in published maps and institutional affiliations.

\section{References}

1. Lynch HT, de la Chapelle A. Genetic susceptibility to nonpolyposis colorectal cancer. J Med Genet. 1999;36:801-18.

2. Moller P, Seppala T, Bernstein I, Holinski-Feder E, Sala P, Evans DG, et al. Cancer incidence and survival in Lynch syndrome patients receiving colonoscopic and gynaecological surveillance: first report from the prospective Lynch syndrome database. Gut. 2017;66:464-72.

3. Mangold E, Pagenstecher C, Friedl W, Mathiak M, Buettner R, Engel C, et al. Spectrum and frequencies of mutations in MSH2 and MLH1 identified in 1,721 German families suspected of hereditary nonpolyposis colorectal cancer. Int $\mathbf{J}$ Cancer. 2005;116:692-702.

4. Thompson BA, Spurdle AB, Plazzer JP, Greenblatt MS, Akagi K, Al-Mulla F, et al. Application of a 5-tiered scheme for standardized classification of 2,360 unique mismatch repair gene variants in the InSiGHT locus-specific database. Nat Genet. 2014;46:107-15.

5. Peltomaki P, Vasen H. Mutations associated with HNPCC predisposition-update of ICG-HNPCC/INSiGHT mutation database. Dis Markers. 2004;20:269-76.

6. Vargas-Parra GM, Gonzalez-Acosta M, Thompson BA, Gomez C, Fernandez A, Damaso E, et al. Elucidating the molecular basis of MSH2-deficient tumors by combined germline and somatic analysis. Int J Cancer. 2017;141:1365-80.

7. Borras E, Pineda M, Brieger A, Hinrichsen I, Gomez C, Navarro $\mathrm{M}$, et al. Comprehensive functional assessment of MLH1 variants of unknown significance. Hum Mutat. 2012;33:1576-88.

8. Drost M, Zonneveld JB, van Hees S, Rasmussen LJ, Hofstra RM, de Wind N. A rapid and cell-free assay to test the activity of Lynch syndrome-associated MSH2 and MSH6 missense variants. Hum Mutat. 2012;33:488-94.

9. Hardt K, Heick SB, Betz B, Goecke T, Yazdanparast H, Kuppers $\mathrm{R}$, et al. Missense variants in hMLH1 identified in patients from the German HNPCC consortium and functional studies. Fam Cancer. 2011;10:273-84.

10. Plotz G, Welsch C, Giron-Monzon L, Friedhoff P, Albrecht M, Piiper A, et al. Mutations in the MutSalpha interaction interface of MLH1 can abolish DNA mismatch repair. Nucleic Acids Res. 2006;34:6574-86

11. Auclair J, Busine MP, Navarro C, Ruano E, Montmain G, Desseigne F, et al. Systematic mRNA analysis for the effect of MLH1 and MSH2 missense and silent mutations on aberrant splicing. Hum Mutat. 2006;27:145-54.

12. Lastella P, Surdo NC, Resta N, Guanti G, Stella A. In silico and in vivo splicing analysis of MLH1 and MSH2 missense mutations shows exon- and tissue-specific effects. BMC Genomics. 2006;7:243.

13. van der Klift HM, Jansen AM, van der Steenstraten N, Bik EC, Tops CM, Devilee P, et al. Splicing analysis for exonic and intronic mismatch repair gene variants associated with Lynch syndrome confirms high concordance between minigene assays and patient RNA analyses. Mol Genet Genomic Med. 2015;3:327-45.

14. Yamaguchi T, Wakatsuki T, Kikuchi M, Horiguchi SI, Akagi K. The silent mutation MLH1 c.543C > T resulting in aberrant splicing can cause Lynch syndrome: a case report. Jpn J Clin Oncol. 2017;47:576-80.

15. Baehring J, Sutter C, Kadmon M, Doeberitz MV, Gebert J. A 'nonsense' mutation leads to aberrant splicing of hMLH1 in a German hereditary non-polyposis colorectal cancer family. Fam Cancer. 2006;5:195-9.

16. Nystrom-Lahti M, Holmberg M, Fidalgo P, Salovaara R, de la Chapelle A, Jiricny J, et al. Missense and nonsense mutations in codon 659 of MLH1 cause aberrant splicing of messenger RNA in HNPCC kindreds. Genes Chromosomes Cancer. 1999;26:372-5.

17. Stella A, Wagner A, Shito K, Lipkin SM, Watson P, Guanti G, et al. A nonsense mutation in MLH1 causes exon skipping in three unrelated HNPCC families. Cancer Res. 2001;61:7020-4.

18. Cartegni L, Krainer AR. Correction of disease-associated exon skipping by synthetic exon-specific activators. Nat Struct Biol. 2003;10:120-5. 
19. Soret J, Gabut M, Tazi J. SR proteins as potential targets for therapy. Prog Mol Subcell Biol. 2006;44:65-87.

20. Charbonnier F, Martin C, Scotte M, Sibert L, Moreau V, Frebourg T. Alternative splicing of MLH1 messenger RNA in human normal cells. Cancer Res. 1995;55:1839-41.

21. Clarke LA, Jordan P, Boavida MG. Cell type specificity in alternative splicing of the human mismatch repair gene $\mathrm{hMSH} 2$. Eur J Hum Genet. 2000;8:347-52.

22. Genuardi M, Viel A, Bonora D, Capozzi E, Bellacosa A, Leonardi F, et al. Characterization of MLH1 and MSH2 alternative splicing and its relevance to molecular testing of colorectal cancer susceptibility. Hum Genet. 1998;102:15-20.

23. Takahashi R, Nagai K. Differences in expression between transcripts using alternative promoters of hMLH1 gene and their correlation with microsatellite instability. Oncol Rep. 2009;22:265-71.

24. Spurdle AB, Couch FJ, Hogervorst FB, Radice P, Sinilnikova OM. Group IUGVW: prediction and assessment of splicing alterations: implications for clinical testing. Hum Mutat. 2008;29:1304-13.

25. Vreeswijk MP, van der Klift HM. Analysis and interpretation of RNA splicing alterations in genes involved in genetic disorders. Methods Mol Biol. 2012;867:49-63.

26. Modrek B, Resch A, Grasso C, Lee C. Genome-wide detection of alternative splicing in expressed sequences of human genes. Nucleic Acids Res. 2001;29:2850-9.

27. Thompson BA, Martins A, Spurdle AB. A review of mismatch repair gene transcripts: issues for interpretation of mRNA splicing assays. Clin Genet. 2015;87:100-8.

28. Davy G, Rousselin A, Goardon N, Castera L, Harter V, Legros A, et al. Detecting splicing patterns in genes involved in hereditary breast and ovarian cancer. Eur J Hum Genet. 2017;25:1147-54.

29. Houdayer C, Caux-Moncoutier V, Krieger S, Barrois M, Bonnet F, Bourdon V, et al. Guidelines for splicing analysis in molecular diagnosis derived from a set of 327 combined in silico/in vitro studies on BRCA1 and BRCA2 variants. Hum Mutat. 2012;33:1228-38.

30. Lewis BP, Green RE, Brenner SE. Evidence for the widespread coupling of alternative splicing and nonsense-mediated mRNA decay in humans. Proc Natl Acad Sci USA. 2003;100:189-92.

31. Andreutti-Zaugg C, Scott RJ, Iggo R. Inhibition of nonsensemediated messenger RNA decay in clinical samples facilitates detection of human MSH2 mutations with an in vivo fusion protein assay and conventional techniques. Cancer Res. 1997;57:3288-93.

32. Umar A, Boland CR, Terdiman JP, Syngal S, de la Chapelle A, Ruschoff $\mathrm{J}$, et al. Revised Bethesda guidelines for hereditary nonpolyposis colorectal cancer (Lynch syndrome) and microsatellite instability. J Natl Cancer Inst. 2004;96:261-8.

33. Morak M, Koehler U, Schackert HK, Steinke V, Royer-Pokora B, Schulmann $\mathrm{K}$, et al. Biallelic MLH1 SNP cDNA expression or constitutional promoter methylation can hide genomic rearrangements causing Lynch syndrome. J Med Genet. 2011;48:513-9.

34. Morak M, Schackert HK, Rahner N, Betz B, Ebert M, Walldorf C, et al. Further evidence for heritability of an epimutation in one of 12 cases with MLH1 promoter methylation in blood cells clinically displaying HNPCC. Eur J Hum Genet. 2008;16:804-11.

35. Holinski-Feder E, Muller-Koch Y, Friedl W, Moeslein G, Keller $\mathrm{G}$, Plaschke J, et al. DHPLC mutation analysis of the hereditary nonpolyposis colon cancer (HNPCC) genes hMLH1 and hMSH2. J Biochem Biophys Methods. 2001;47:21-32.

36. van der Klift HM, Tops CM, Hes FJ, Devilee P, Wijnen JT. Insertion of an SVA element, a nonautonomous retrotransposon, in PMS2 intron 7 as a novel cause of Lynch syndrome. Hum Mutat. 2012;33:1051-5.
37. Sumitsuji I, Sugano K, Matsui T, Fukayama N, Yamaguchi K, Akasu T, et al. Frequent genomic disorganisation of MLH1 in hereditary non-polyposis colorectal cancer (HNPCC) screened by RT-PCR on puromycin treated samples. J Med Genet. 2003;40: e30.

38. Etzler J, Peyrl A, Zatkova A, Schildhaus HU, Ficek A, Merkelbach-Bruse S, et al. RNA-based mutation analysis identifies an unusual MSH6 splicing defect and circumvents PMS2 pseudogene interference. Hum Mutat. 2008;29:299-305.

39. Renkonen E, Lohi H, Jarvinen HJ, Mecklin JP, Peltomaki P. Novel splicing associations of hereditary colon cancer related DNA mismatch repair gene mutations. J Med Genet. 2004;41:e95.

40. Renkonen E, Zhang Y, Lohi H, Salovaara R, Abdel-Rahman WM, Nilbert M, et al. Altered expression of MLH1, MSH2, and MSH6 in predisposition to hereditary nonpolyposis colorectal cancer. $\mathrm{J}$ Clin Oncol. 2003;21:3629-37.

41. Nomura S, Sugano K, Kashiwabara H, Taniguchi T, Fukayama N, Fujita S, et al. Enhanced detection of deleterious and other germline mutations of hMSH2 and hMLH1 in Japanese hereditary nonpolyposis colorectal cancer kindreds. Biochem Biophys Res Commun. 2000;271:120-9.

42. Pagenstecher C, Wehner M, Friedl W, Rahner N, Aretz S, Friedrichs N, et al. Aberrant splicing in MLH1 and MSH2 due to exonic and intronic variants. Hum Genet. 2006;119:9-22.

43. Tournier I, Vezain M, Martins A, Charbonnier F, BaertDesurmont S, Olschwang S, et al. A large fraction of unclassified variants of the mismatch repair genes MLH1 and MSH2 is associated with splicing defects. Hum Mutat. 2008;29:1412-24.

44. Whiley PJ, de la Hoya M, Thomassen M, Becker A, Brandao R, Pedersen IS, et al. Comparison of mRNA splicing assay protocols across multiple laboratories: recommendations for best practice in standardized clinical testing. Clin Chem. 2014;60:341-52.

45. Davoodi-Semiromi A, Lanyon GW, Davidson R, Connor MJ. Aberrant RNA splicing in the hMSH2 gene: molecular identification of three aberrant RNA in Scottish patients with colorectal cancer in the West of Scotland. Am J Med Genet. 2000;95:49-52.

46. Mori Y, Shiwaku H, Fukushige S, Wakatsuki S, Sato M, Nukiwa $\mathrm{T}$, et al. Alternative splicing of hMSH2 in normal human tissues. Hum Genet. 1997;99:590-5.

47. Palmirotta R, Veri MC, Curia MC, Aceto G, D'Amico F, Esposito DL, et al. Transcripts with splicings of exons 15 and 16 of the hMLH1 gene in normal lymphocytes: implications in RNA-based mutation screening of hereditary non-polyposis colorectal cancer. Eur J Cancer. 1998;34:927-30.

48. Rhine CL, Cygan KJ, Soemedi R, Maguire S, Murray MF, Monaghan SF, et al. Hereditary cancer genes are highly susceptible to splicing mutations. PLoS Genet. 2018;14:e1007231.

49. Caux-Moncoutier V, Pages-Berhouet S, Michaux D, Asselain B, Castera L, De Pauw A, et al. Impact of BRCA1 and BRCA2 variants on splicing: clues from an allelic imbalance study. Eur J Hum Genet. 2009;17:1471-80.

50. Hesson LB, Packham D, Kwok CT, Nunez AC, Ng B, Schmidt C, et al. Lynch syndrome associated with two MLH1 promoter variants and allelic imbalance of MLH1 expression. Hum Mutat. 2015;36:622-30.

51. Wei Q, Bondy ML, Mao L, Gaun Y, Cheng L, Cunningham J, et al. Reduced expression of mismatch repair genes measured by multiplex reverse transcription-polymerase chain reaction in human gliomas. Cancer Res. 1997;57:1673-7.

52. Perera S, Bapat B. The MLH1 variants p.Arg265Cys and p. Lys618Ala affect protein stability while p.Leu749Gln affects heterodimer formation. Hum Mutat. 2008;29:332.

53. Raevaara TE, Korhonen MK, Lohi H, Hampel H, Lynch E, Lonnqvist KE, et al. Functional significance and clinical 
phenotype of nontruncating mismatch repair variants of MLH1. Gastroenterology. 2005;129:537-49.

54. Hinrichsen I, Brieger A, Trojan J, Zeuzem S, Nilbert M, Plotz G. Expression defect size among unclassified MLH1 variants determines pathogenicity in Lynch syndrome diagnosis. Clin Cancer Res. 2013;19:2432-41.
55. Koger N, Paulsen L, Lopez-Kostner F, Della Valle A, Vaccaro CA, Palmero EI, et al. Evaluation of MLH1 variants of unclear significance. Genes Chromosomes Cancer. 2018;57:350-8.

56. Hayward BE, De Vos M, Valleley EM, Charlton RS, Taylor GR, Sheridan E, et al. Extensive gene conversion at the PMS2 DNA mismatch repair locus. Hum Mutat. 2007;28:424-30. 